\title{
The enhancement of cartilage regeneration by use of a chitosan-based scaffold in a 3D model of microfracture in vitro: a pilot evaluation
}

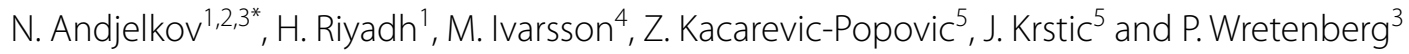

\begin{abstract}
Purpose: Even though various types of scaffolds have been used lately as a complement to microfracture, the exact mechanism of reported cartilage repair improvement when using scaffolds is still unclear. In this study, an effort has been made to identify the specific effects that scaffolds may have on the cells of reparation when using this technique.
\end{abstract}

Methods: A 3-D model in vitro, representing microfracture and containing both chondrocytes and bone marrowderived cells in different experimental conditions was made, and the cells were cultured for eight weeks. Subsequently, the constructs containing our 3-D model were removed from the cell culture medium, fixed in paraffin and analyzed with immunohistochemistry.

Results: Bone marrow - derived cells migrated to the upper compartment of the construct through a perforated nylon membrane containing both enzymatically digested-and non-digested particulated cartilage. The histological sections were stained with hematoxylin, eosin, S-100, SOX-9, Gomori, and procollagen type I and II. When minced cartilage wasn't pretreated with collagenase, exclusively bone-derived cells have created new extracellular matrix as showed by the histological analysis.

Conclusions: In this model of microfracture, bone-derived cells but not chondrocytes have shown to have an active role in new cartilage formation without predigestion with collagenase. Moreover, it seems that the addition of a chitosan-based scaffold may lead to the improvement of a new cartilage matrix synthesis and integration. This effect hasn't been seen without the use of scaffold or when a fibrin- or a collagen-based scaffold have been used.

\section{Introduction}

Even though microfracture as a technique has been in use for a long time [29], a discussion about the exact mechanism for cartilage repair during this technique and cartilage repair techniques derived from it is still open [10]. This technique will not be more thoroughly described here since it is the best known and most widely

\footnotetext{
*Correspondence: nenad.andjelkov@dbivard.se

1 Department of Orthopedics, Västmanlands Regional Hospital, Västerås, Sweden

Full list of author information is available at the end of the article
}

used cartilage repair procedure at the present. What's important to mention here is that it's based on the bone marrow stimulation using mesenchymal stromal cells (MSCs) from the subchondral bone for regeneration [29]. A variety of matrix-assisted microfracture techniques have been in use since the original technique was developed [9, 22]. The AMIC (Assisted Matrix Induced Chondrogenesis) technique is one of those scaffold-based techniques which was derived from the microfracture [21]. It has been known that these techniques may lead to the creation of hyaline and fibrocartilage [11] but the knowledge about what exactly happens on the cellular 


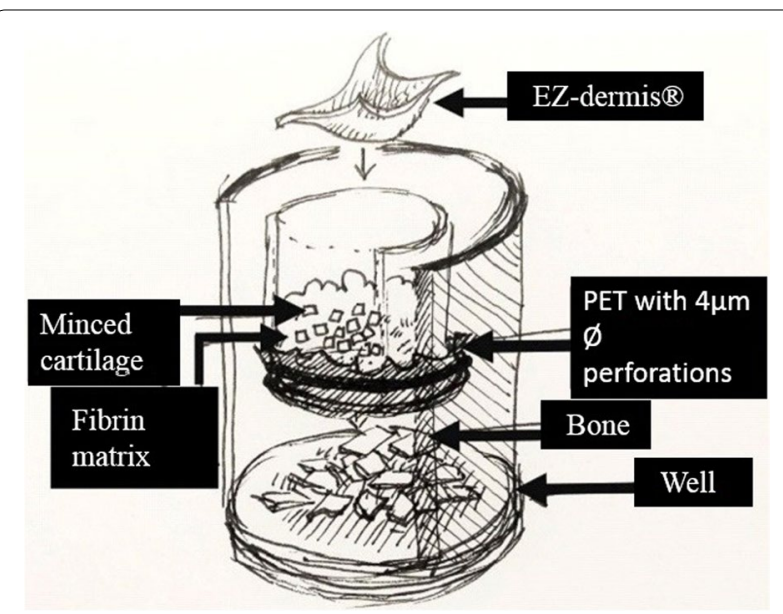

Fig. 1 Microfracture 3D - model used in our experimental setup in vitro: EZ Derm ${ }^{\circledR}$ - porcine epidermis. Minced cartilage. Fibrin matrix - in some groups it has been replaced with chitosan-based matrix. PET membrane with $4 \mu \mathrm{m}$ pores and perforated with BD Microlance ${ }^{\circledR}$ $21 \mathrm{~g} \times 1 "-0,8 \times 25 \mathrm{~mm}$ needle. Bone chips at the bottom of plastic well and tissue level during the repair process itself is still insufficient.

The main goals of the study were to detect the basic processes like cell migration and differentiation, extracellular matrix formation and expression of some crucial bio-markers of chondrogenesis depending on different experimental conditions - absence or presence of three different types of scaffolds, i.e. fibrin, collagen-based and chitosan-based scaffold. The method used was procollagen type I, procollagen type II, and SOX-9 detection as one of the crucial biomarkers of fibrocartilage, hyaline cartilage and chondrogenesis respectively [3, 30]. Gomori's staining for detection of extracellular cartilage matrix formation has also been used [8]. The main study hypothesis was that the use of the scaffolds would improve all the parameters mentioned above.

\section{Materials and methods}

A 3-D model in vitro that should represent the microenvironment that is created by microfracturing (Fig. 1) was made and the cells were cultured for eight weeks in different experimental conditions. Subsequently, the

Table 1 Eight weeks of culture in CellCrown ${ }^{\circledR}$. Perforated PET membrane in all groups. Some groups weren't pretreated with collagenase while the others were predigested with both low and high enzyme concentrations. Some groups didn't contain any scaffold, some groups contained fibrin, some groups EZ-derm ${ }^{\circledR}$, while other groups contained chitosanbased scaffold. Upper compartment with PET membrane was fixated, emerged in paraffin and histology sections were made. Hematoxylin, eosin and trichrome staining was done. Immunohistochemistry analysis for four markers was performed: procollagen type I and II, SOX-9 and S-100

\begin{tabular}{|c|c|c|c|c|c|c|c|}
\hline Well & Plate & Bone & Cartilage & Collagenase (conc.) & Fibrin & EZ-derm & Chitosan \\
\hline 1 & A & + & + & - & - & - & + \\
\hline 2 & A & + & + & - & - & + & - \\
\hline 3 & A & + & + & - & - & + & - \\
\hline 4 & A & + & + & - & + & + & - \\
\hline 5 & A & + & + & - & - & + & - \\
\hline 6 & A & + & + & - & + & + & - \\
\hline 7 & A & + & + & - & - & + & - \\
\hline 8 & B & + & + & Low & - & - & + \\
\hline 9 & B & + & + & Low & - & + & - \\
\hline 10 & B & + & + & Low & + & + & - \\
\hline 11 & B & + & + & Low & - & + & - \\
\hline 12 & B & + & + & Low & + & + & - \\
\hline 13 & B & + & + & Low & - & + & - \\
\hline 14 & B & + & + & High & - & - & + \\
\hline 15 & B & + & + & High & - & + & - \\
\hline 16 & B & + & + & High & + & + & - \\
\hline 17 & B & + & + & High & - & + & - \\
\hline 18 & B & + & + & High & + & + & - \\
\hline 19 & B & + & + & High & - & + & - \\
\hline 20 & B & - & + & High & - & + & - \\
\hline 21 & $A$ & + & - & - & - & + & - \\
\hline
\end{tabular}


constructs containing a 3-D model were removed from the cell culture medium, fixed in paraffin and analyzed with immunohistochemistry.

\section{Culture of cartilage in a 3-D model}

Cartilage and trabecular bone were obtained from the discarded femoral heads during hip arthroplasty (two patients, both women, Pat. No. 1, 70 years of age, Pat. No 2, 69 years of age, both with osteoarthritis grade II-IV), as well as from the patients undergoing total knee replacement surgery, where macroscopically normal cartilage could be found in the lateral femoral condyle area (two patients, both women, Pat. No. 3, 76 years of age, Pat. No 4, 75 years of age). The specimens from all four patients were collected and the experiments were repeated for each of them. The cartilage was cut into small pieces $\left(1-2 \mathrm{~mm}^{3}\right)$ and the trabecular bone was cut into bone chips small enough to fit the wells and the space under the inserts, and then incubated in different experimental settings according to Table 1 , some of them without and some of them with 20 units/ml collagenase II, respectively 100 units/ml collagenase II (Sigma-Aldrich, Missouri, USA) in Dulbecco's modified eagle medium (DMEM) nutrient solution (ThermoFisher, Waltham, Massachusetts, USA) at $37^{\circ} \mathrm{C}$ for $16 \mathrm{~h}$ under gentle movement. The samples were centrifuged at $260 \mathrm{xg}$ for five minutes and washed in $10 \mathrm{ml}$ phosphate buffered saline (PBS) (ThermoFisher, Waltham, Massachusetts, USA) followed by centrifuged for $260 \mathrm{xg}$ for five minutes. The final pellet was then placed in a special insert containing $8-\mu \mathrm{m}$ PET or 1.2- $\mu \mathrm{m}$ nylon membrane (Fig. 1; CellCrown ${ }^{\mathrm{TM}} 24$, Cat. Nr. C70001F, C12001F, Scaffdex, Tampere, Finland) perforated with a sterile $0.6 \times 25-\mathrm{mm}$ needle. In this model, this upper compartment should represent the cartilage defect that could be found in microfracture after debridement of cartilage injury. The needle perforations through the membranes in the bottom of the inserts represented the holes that are made in the subchondral bone during microfracture. The fibrin matrix, replacing natural fibrin that could be found in the "super clot" [29] post microfracture (TISSEEL DUO QUICK, Baxter, Sweden) was then added covering in total cartilage fragments, and the inserts were placed onto the top of the cancellous bone biopsies previously situated in a sterile 24 -well plate. In this model, the cancellous bone chips represented the subchondral bone in microfracture and could easily be separated from the inserts at the end of cell culturing and prior to inserts analysis. Finally, some inserts were left without any scaffolds, in some of them as previously mentioned, fibrin was added, while some inserts were filled with chitosan-based scaffold made by this research group and some were covered with EZ Derm ${ }^{\circledR}$ - porcine skin consisting of a superficial thin layer mainly made of dead cells - epidermis, and another much thicker layer - dermis consisting mainly of collagen fibers, which should replace commercially available collagen-based scaffolds currently used in certain cartilage repair techniques. Wells were filled with DMEM supplemented with $20 \%$ fetal bovine serum (FBS) and antibiotics, so called "growth medium" that has been changed after a week with a DMEM supplemented with $10 \%$ serum. The nutrient solution was changed twice a week for eight weeks with DMEM and 10\% FBS. An overview of the contents of the wells is shown in Table 1.

\section{Fixation and dehydration of cultures}

After 8 weeks of culture, the content of the inserts was taken out and positioned in padded cassettes so that central portions were facing surface after paraffin embedding. Specimens were fixed in 3.7\% formaldehyde. Dehydration was performed with Tissue Processor TPC15DUO (Medite GmbH, Burgdorf, Germany).

\section{Embedding and sectioning}

After dehydration, fixed specimens were embedded in paraffin. Four micrometer tissue sections were generated with Leica RM2255 automatic microtome (Leica biosystems, Mölndal, Sweden). The sections were floated in a cold-water bath and mounted on Super frost ${ }^{\circledR}$ plus gold microscope glass-slides (Fisher Scientific, Gothenburg, Sweden). Slides were placed on a hot plate for one minute. Slides were placed in a $60{ }^{\circ} \mathrm{C}$ heating cabinet for four days.

\section{Hematoxylin and eosin staining}

The sections were rehydrated in xylene/ ethanol/water, stained with Mayer's hematoxylin (Histolab, Gothenburg, Sweden) for five minutes and rinsed with tap water for four minutes. After that, they were stained for one minute in eosin solution (distilled water, $0.25 \%$ eosin, $80 \%$ ethanol, and $90 \mathrm{mM}$ acetic acid) and rinsed with tap water for one minute. Finally, the sections were dehydrated in distilled water/ethanol/xylene and mounted with cover slips and Pertex mounting agent (Histolab, Gothenburg, Sweden).

\section{Gomori's trichrome staining}

Rehydrated sections were stained with Harris hematoxylin for six minutes and rinsed for five minutes with tap water. Sections were stained with Gomori's stain solution A $(0.6 \%$ chromotrope $2 \mathrm{R}, 0.6 \%$ phosphovolvic acid hydrate, $0.3 \%$ Fast Green FCF, $0.17 \mathrm{M}$ acetic acid and distilled water) for ten minutes and rinsed with distilled water. Slides were briefly immersed in Gomori's stain solution B (0.35 M acetic acid and distillate water), dehydrated and mounted. 


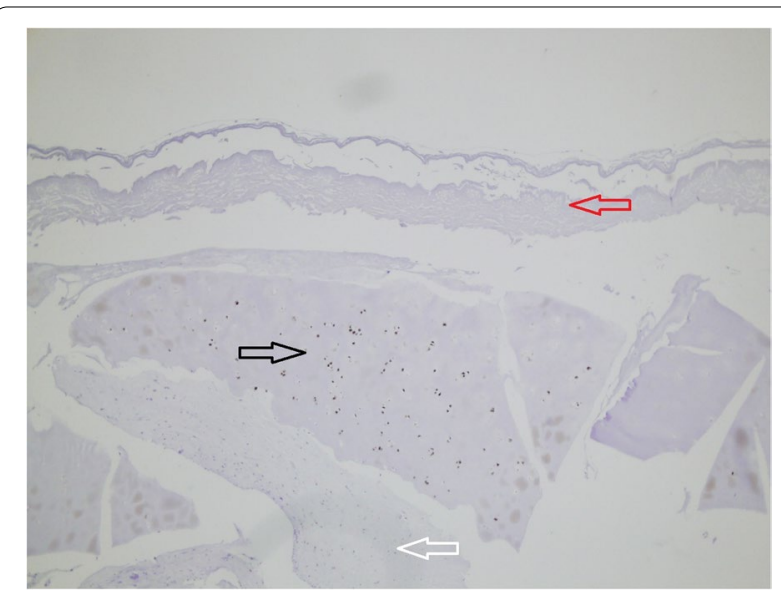

Fig. 2 Minced cartilage + bone chips + fibrin, no digestion with collagenase (Well 4): Positive staining with S-100 for chondrocytes situated in the lacunas, no chondrocytes seen outside the cartilage pieces (marked with black arrow). Cell elements with extracellular matrix beneath the cartilage piece positioned centrally in the figure. These cells weren 't stained with S-100 and supposedly originate from the bone chips, i.e. bone-marrow derived cells (marked with white arrow). In the upper corner porcine epidermis with no cell elements (marked with red arrow) can be seen. No adhesion of the newly formed matrix with the piece of cartilage

\section{Immunohistochemistry}

Immunohistochemistry staining was performed with Dako REAL ${ }^{\mathrm{TM}}$ EnVision ${ }^{\mathrm{TM}}$ Detection System Peroxidase / $\mathrm{DAB}+$, Rabbit / Mouse (Dako, Agilent, Santa Clara, California, USA). Rehydrated sections were washed twice in wash buffer and incubated with Proteinase $\mathrm{K}$ for ten minutes. Thereafter, they were rinsed twice with wash buffer and incubated with primary antibodies S100 (ThermoFisher, Waltham, Massachusetts, USA), procollagen II (Abcam, Cambridge, UK) or Sox9 (Abcam, Cambridge, UK), diluted 1: 500 for $30 \mathrm{~min}$. The sections were rinsed then with wash buffer twice and incubated with peroxidase blocking for eight minutes. Next, the sections were rinsed with wash buffer twice and incubated with EnVision complex for $30 \mathrm{~min}$. Sections were rinsed twice with washed buffer and incubated with Chromium (DAB) for $15 \mathrm{~min}$. Sections were rinsed with wash buffer twice and rinsed in running tap water for $5 \mathrm{~min}$. Finally, they were stained with Mayer's hematoxylin for ten seconds and rinsed with tap water for five minutes. After dehydration, slides were mounted with Pertex mounting agent.

\section{Results}

\section{Culture of cartilage in a 3-D model}

Bone marrow-derived cells migrated to the upper compartment of the construct through a perforated nylon membrane containing either enzymatically digested- or non-digested particulated cartilage. If not predigested

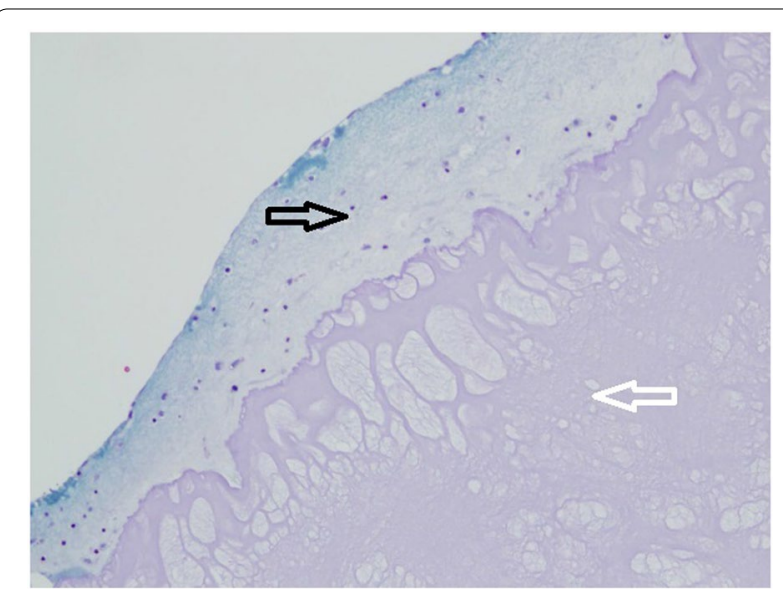

Fig. 3 New cartilage formation in the upper part of the photo detected with Gomori staining and visualized in blue (marked with black arrow, well 8). The cell elements situated within the newly formed cartilage matrix. Chitosan based scaffold served as a matrix in the lower compartment stained in pink (marked with white arrow). God adhesion of the newly formed extracellular matrix to the scaffold

with collagenase, the chondrocytes weren't able to escape the matrix and remained within the cartilage pieces.

\section{Hematoxylin and eosin staining}

Bone-marrow derived cells have migrated towards minced cartilage (Fig. 2), New, extracellular matrix was created outside the non-digested cartilage pieces (Fig. 2). Without cellular elements in the upper compartment, i.e. chondrocytes, but only EZ-derm, no bone-marrow

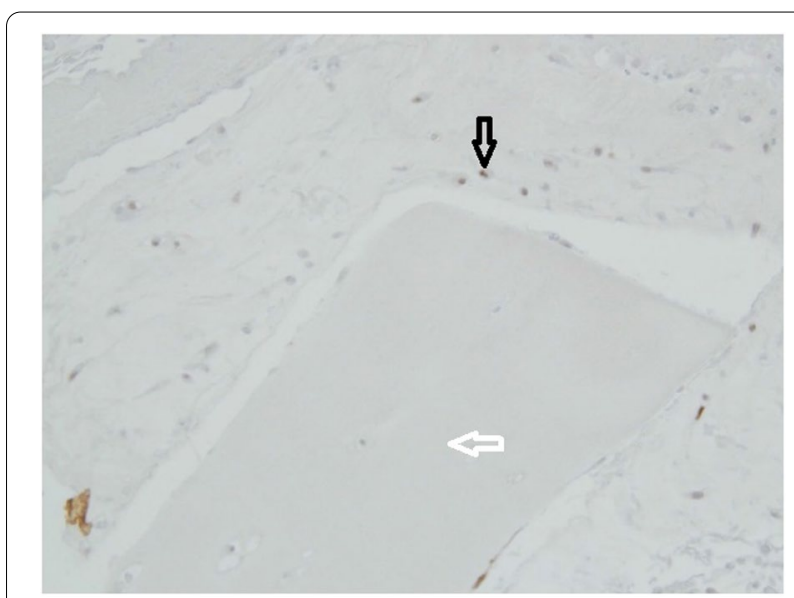

Fig. 4 Some week positive signals for SOX-9 stained cells in the newly formed cartilage matrix from the digested particulated cartilage (marked with black arrow, well 10). Minced cartilage in the middle (marked with white arrow). Poor adhesion of the newly formed matrix with the piece of cartilage 


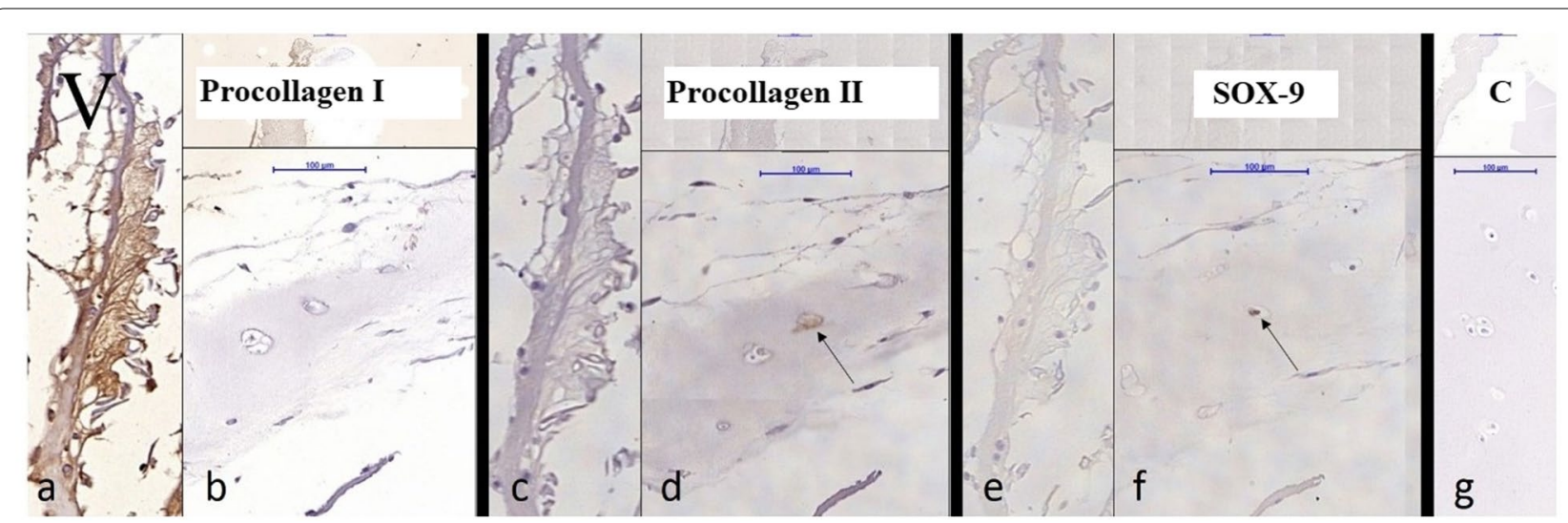

Fig. 5 Cartilage cultivation containing cartilage pieces treated with $100 \mathrm{U} / \mathrm{ml}$ collagenase and cultured with fibrin matrix (Well 16). a and b: Fibrin respectively cartilage. Figure a representing immunohistochemistry with antibody against procollagen type I that was non-specific and $\mathbf{b}$ representing positive staining within cartilage. $\mathbf{c}$ and $\mathbf{d}$ : Fibrin respectively cartilage. Negative respectively positive immunohistochemistry with antibody against procollagen type II. e and $\mathbf{f}$ : Fibrin respectively cartilage. Negative respectively positive immunochemistry with antibody against sex-determining region Y-related high-mobility-group box 9 (SOX-9). g: Negative control for SOX-9

derived cells migration were noticed towards or through the membrane.

\section{Gomori's trichrome staining}

A collagen-specific extracellular matrix next to the chitosan-based scaffold was confirmed with trichrome (Gomori) staining (Fig. 3). The attempts to show positive staining with trichrome failed in all other experimental groups with the exception of non-digested cartilage pieces, which were stained as expected.

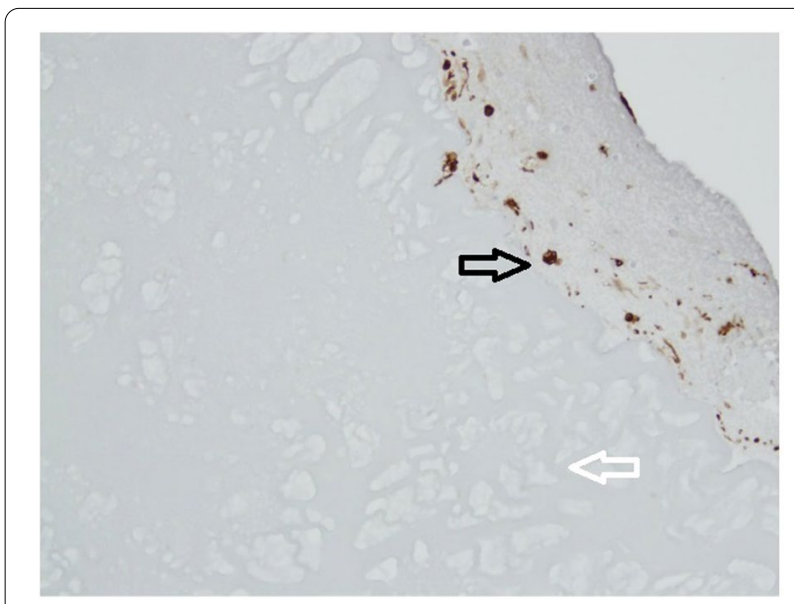

Fig. 6 Immunohistochemistry with S-100 (Well 8). Scaffold in the lower corner (marked with white arrow), newly formed cartilage matrix in the upper corner (marked with black arrow). Sporadic staining with S-100 at the periphery of the newly formed cartilage matrix and more abundant signals in the newly formed cartilage next to the scaffold (marked with black arrow). God adhesion of the newly formed extracellular matrix to the scaffold

\section{Immunohistochemistry}

Chondrocytes were identified by staining with S-100 and bone derived cells were remained unstained (Fig. 2). Bone-derived cells and not chondrocytes have created new extracellular matrix in the groups without cartilage digestion as showed by S-100 staining (Fig. 2). When predigested with collagenase, chondrocytes from minced cartilage have formed new cartilage matrix as well (Fig. 4). SOX-9 staining was proved to be positive both inside the cartilage pieces (Fig. 5) and with less intensity in the newly created cartilage matrix (Fig. 4). In the last case, chondrocytes within the non-digested cartilage pieces remained unstained with SOX-9 (Fig. 4). Staining with procollagen type II was positive inside of minced cartilage (Fig. 6d), but not outside of it (Fig. 5c). The antibody for procollagen type I gave a strong non-specific staining, the reason why it couldn't be used for further analysis (Fig. 5a and b).

\section{Discussion}

The main findings of this study are the observations related to the use of different type of scaffolds on basic processes like cell migration, differentiation and creation of new cartilage matrix. We have seen in this study the differences in these important processes depending on the absence of any scaffold or the use of various type of scaffolds, namely fibrin, collagen-based or/and chitosanbased scaffold. At present, there are numerous reports of the improvement of cartilage repair with the use of various types of scaffolds [2, 22], while other reports show no such effect $[12,16]$. Most of those studies have been conducted as clinical trials $[16,22]$, giving us the objective measures such as histology and clinical scores, which 
naturally is of greatest interest. Numerous animal studies have been conducted as well, prior to the use of these scaffolds in humans $[12,32]$, providing us with the histology of the repaired tissue when using different scaffolds or without them [12, 32]. However, the studies on the basic level, preceding both animal models and clinical trials are not abundant and the authors of this article have failed to find them in the literature. Therefore, this 3 -D model in vitro of microfracture, presented here can be seen as an attempt to add some new knowledge to this topic and would hopefully lead to the improvement of the scaffolds used in matrix-assisted microfracture techniques prior to their clinical use and subsequently the improvement of clinical outcomes as well.

\section{Scaffold-free experimental setup}

The most relevant observation when not having any scaffold in the upper compartment has been that cellular migration towards and within upper compartment hasn't been noticed. This finding is somehow expected since it has been known that the fibroblast-like cells that are phenotypically similar to MSCs [7] when in culture may need chemo-tactical, mechano-tactical and electro-tactical factors for cell migration $[6,25]$. This would be one of the main limitations of this model since it has been known that even without the scaffold, the space within debrided cartilage is almost immediately filled with blood, growth factors and fibrin, a so called "super clot" formation [29].

Another limitation of this model that should be mentioned and discussed at this point is the use of collagenase in certain experimental groups. In this 3-D model of microfracture in vitro bone-derived cells and not chondrocytes have shown to have an active role in new cartilage formation when minced cartilage haven't been pretreated with collagenase. However, when digested with collagenase, chondrocytes alone from particulated cartilage have created a neocartilage formation. Under normal circumstances, the predigestion with collagenase during microfracture procedure doesn't occur. Since it has been known from a previous study from this group [1] that the chondrocytes wouldn't be able to escape the matrix without the enzymatic digestion, the collagenase has been used. This finding has been confirmed even in this study since no outgrowth of chondrocytes has been noticed in the non-digested experimental groups (Fig. 2). However, even in a normal, clinical situation in microfrature, a smaller amount of proteolytic enzymes may be present at the site of injury and inflammation [17, 28]. Nonetheless, this concentration is unlikely to be in such high concentrations as those used in the experiments and therefore it doesn't seem likely that chondrocyte relase occurs in microfracture. Consequently, the probability that in a clinical situation during microfracture the enzymatic digestion of cartilage takes place so that "released" chondrocytes can participate actively in the novel cartilage matrix synthesis is very low. Finally, no significant differences have been found for 20 units $/ \mathrm{ml}$ collagenase II, respectively 100 units $/ \mathrm{ml}$ collagenase II.

\section{Fibrin scaffold}

Fibrin has been used widely in cartilage repair procedures both alone as a scaffold [31] or as a glue to secure the scaffold in place post surgery [24]. In the experimental model applied in this study, it hasn't been seen any migration of the cells into the fibrin matrix and therefore no new extracellular matrix formation inside. Similarly, very poor or no integration to other scaffolds and extracellular matrices has been noticed (Figs. 2 and 4). A commercially available fibrin preparation has been used in this study, very similar to those routinely used for matrix fixation during matrix-assisted microfracture techniques [27]. Despite the mechanical equivalency, this fibrin preparation differs in some characteristics from the autologous one that may be present in the normal clinical situation in microfracture [14].

\section{Collagen-based scaffold}

As for the collagen-based matrices, the explanation has already been given in the "Materials and Methods" section for the choice of use of EZ-derm ${ }^{\circledR}$ as a substitute for a collagen-based matrix. Indeed, this type of scaffolds has been widely used in so-called matrix-assisted microfracture techniques [16]. By its structure, the EZ-derm ${ }^{\circledR}$ is almost identical to the one of most commonly used matrices in cartilage repair which has also been derived from the porcine skin - Chondro- Gide ${ }^{\circledR}$ [27]. In the histological sections obtained in this study, scarce or no cell migration has been seen towards or into the EZ-derm ${ }^{\circledR}$ (Figs. 1 and 2). Subsequently, no extracellular matrix production has been identified next to it (Fig. 2). The poor integration of EZ-derm ${ }^{\circledR}$ with the other components of the construct has been seen as well (Fig. 2). In this experimental group, the new cartilage matrix has been synthesized separately and beneath the EZ-derm ${ }^{\circledR}$ (Fig. 2). As previously mentioned, chondrocytes within minced cartilage have been stained with S-100 (Fig. 2) as a rule. The staining with procollagen type II has been positive within minced cartilage and so far, hasn't been detected outside of it, in the newly formed extracellular matrix to be more specific (Fig. $5 \mathrm{c}$ and d). At the present, the conclusion that can be made from these findings is that the chondrocytes from minced cartilage are metabolically active and are still producing new collagen type II after 8 weeks in culture $[4,13]$. As said previously, the staining with procollagen type I was unspecific and therefore these results can't be used for any further analysis (Fig. $5 a$ and b). Future 
immunohistochemistry for both procollagen type I and II is recommended for better understanding of their expression dynamics and distribution in newly created extracellular matrix. This with concern to the fact that collagen type I is primarily expressed in fibrocartilage [19] while collagen type II is the crucial marker of hyaline-like cartilage [33]. That is why it is very important to further examine the expression of these two biomarkers, specifically in relation to their presence in scaffolds or not. The staining with SOX-9 has mainly been detected in cartilage pieces (Fig. 5f). At the same time, weak SOX-9 staining has been noticed in the newly formed cartilage matrix (Fig. 4). This matrix has been synthetized from the chondrocytes originated from digested particulated cartilage. According to the previous reports the SOX-9 gene is expressed from the multipotent skeletal progenitor stage and is active throughout chondrocyte differentiation [18]. It is repressed in hypertrophic chondrocytes in cartilage growth plates but remains expressed throughout life in permanent chondrocytes of healthy articular cartilage [18]. The absence of SOX-9 expression in matrix in most of the cases therefore suggests the presence of cells other than chondrocytes in these sections, most probably bonemarrow derived cells. Reportedly, MSCs become hypertrophic in long term despite chondrogenic differentiation following the pathway of growth plate chondrocytes [26]. All together, by failing to prove both the markers of chondrogenesis (SOX-9, procollagen type I and II), the marker of adult chondrocyte phenotype (S-100) in the cell elements within the newly synthetized extracellular matrix, and cartilage-like nature of that matrix (trichrome/Gomori staining), the chondrogenic potential of the collagen-based scaffold in this experimental model hasn't been confirmed. In addition to that, as previously said very poor integration with other components of the insert, as well as scarce cell adhesion have been detected.

\section{Chitosan-based scaffold}

Chitosan has been routinely used as a scaffold in cartilage repair [22]. Some very good clinical data have been reported on its use [22]. In this model, similarly as in the case of collagen-based scaffolds, a substitute to the commercially available scaffold has been used. This time, the scaffold used was made by this research group. At this point, a further description of the scaffold characteristics is unfortunately not possible due to patent rights protection.

First of all, an excellent integration of the newly formed cartilage matrix, as proved by Gomori's staining, has been seen (Fig. 3). These two findings speak strongly in favour of the chondrogenic potential of this scaffold. However, only certain cells in the newly formed cartilage matrix have been stained with S-100. These were mainly situated next to the chitosan-based scaffold (Fig. 6). From past studies it has been known that S-100 is expressed in differentiated MSCs but not in an undifferentiated cell form [5]. One explanation could be that chondrocytes have been detected in the newly created extracellular matrix. The other possible explanation could be that not all chondrocytes express S-100 as it has been reported before [20]. In this study, the immunostaining for both S-100 sub units (a and b) was detected in chondrocytes in superficial, intermediate, and deep zones of normal articular cartilage [20]. However, in matrix, only the superficial zone was stained positively [20] In arthritic joints, intense immunostaining was detected in clustered chondrocytes in the hypercellular area, while weak or no immunostaining have been seen in isolated chondrocytes in the hypocellular area of articular cartilage [20].

No staining with SOX-9 has been detected next to the chitosan-based scaffold. SOX-9 is a transcription factor that regulates chondrogenesis and its role in chondrogenic differentiation of MSCs triggered by materials is poorly understood [15]. A study has shown that the role of SOX-9 regulation by materials is like that of growth factors, suggesting that a well-designed scaffold may replace growth factors in chondrogenesis [15]. A correlation between cell viability and SOX-9 downregulation has also been reported [23]. Probably, additional experiments should be done at different times to look for the expression of this and other bio-markers. This could be one of the main flows of this study.

Finally, similar to the case of other scaffolds no or very poor migration of the cells into the depth of the matrix has been seen. However, the preliminary data that hasn't been reported in this study shows scarce cell migration into this matrix by identifying cellular DNA inside of the matrix. Again, not going into details about the matrix structure, the pore size should be enlarged to increase the permeability of the matrix for the cells.

This 3-D model of microfracture in vitro may be seen as a very useful tool for the analysis of the basic cell processes in microfracture and the influence of different scaffolds on the processes leading to cartilage repair. Furthermore, it could serve as a suitable environment for making modifications and improvements in scaffolds prior to their clinical use in the matrix-assisted microfrature techniques. The authors of this text consider that as the main clinical relevance and contribution of this 3-D'model. Further studies should be conducted for better understanding of the dynamics of cartilage biomarkers expression in microfracture and the influence of different types of scaffolds and their adjustments on this expression and neocartilage formation.

\footnotetext{
Acknowledgements

No other contributors to list here.
} 
No external sources of funding.

The study has been done with the funding from University of Örebro.

\section{Authors' contributions}

Author 1. Has participated in the study design and writing the manuscript. Has collected the biopsies. Author 2. Has participated in the study design and writing the manuscript. Author 3. Has participated in the study design and writing the manuscript. Has performed the experiments. Author 4. Has participated in the scaffold synthesis and writing the manuscript. Author 5. Has participated in the scaffold synthesis and writing the manuscript. Author 6. Has participated in the study design and writing the manuscript. Has collected the biopsies. The author(s) read and approved the final manuscript.

\section{Ethics approval and consent to participate}

The Regional Ethical Review Board in Uppsala, at Uppsala University has approved the study, and informed consent was obtained from all the patients donating their cartilage and bone specimens for the study. The number of ethics approval is 2009/092.

\section{Competing interests}

The authors declare that they have no conflict of interests.

\section{Author details}

${ }^{1}$ Department of Orthopedics, Västmanlands Regional Hospital, Västerås, Sweden. ${ }^{2}$ Centre for Clinical Research, Uppsala University, Västmanlands Regional Hospital, Västerås, Sweden. ${ }^{3}$ Department of Orthopaedics, School of Medical Sciences, Örebro University, Örebro, Sweden. ${ }^{4}$ Department of Health Sciences, University of Örebro, Örebro, Sweden. ${ }^{5}$ Department of Radiation Chemistry and Physics, Vinca Institute of Nuclear Sciences, University of Belgrade, Belgrade, Serbia.

Received: 27 October 2020 Accepted: 18 January 2021

Published online: 18 February 2021

\section{References}

1. Andjelkov N, Hamberg H, Bjellerup P (2016) No outgrowth of chondrocytes from non-digested particulated articular cartilage embedded in commercially available fibrin matrix: an in vitro study. J Orthop Surg Res 16(11):23

2. Bark S, Piontek T, Behrens P, Mkalaluh S, Varoga D, Gille J (2014) Enhanced microfracture techniques in cartilage knee surgery: fact or fiction? World J Orthop 5(4):444-9

3. Byung-Hyun C, Jae-Hwan K, Sun-Woong K, Hyun-Jin D, Ju-Woong J, Yon Rak C, Hansoo P, Byung-Soo K, Soo-Hong L (2013) Cartilage tissue formation from dedifferentiated chondrocytes by codelivery of BMP-2 and SOX-9 genes encoding bicistronic vector. Cell Transplant 22(9):1519-1528

4. Cakmak O, Buyuklu F, Yilmaz Z, Sahin Fl, Tarhan E, Ozluoglu LN (2005) Viability of cultured human nasal septum chondrocytes after crushing. Arch Facial Plast Surg 7(6):406-409

5. Chen X, Wang XD, Chen G, Lin WW, Yao J, Gu XS (2006) Study of in vivo differentiation of rat bone marrow stromal cells into schwann cell-like cells. Microsurgery 26(2):111-115

6. Chi PY, Spuul P, Tseng FG, Genot E, Chou CF, Taloni A (2019) Cell migration in microfluidic devices: invadosomes formation in confined environments. Adv Exp Med Biol 1146:79-103

7. Denu RA, Nemcek S, Bloom DD, Goodrich AD, Kim J, Mosher DF, Hematti P (2016) Fibroblasts and mesenchymal stromal/stem cells are phenotypically indistinguishable. Acta Haematol 136(2):85-97

8. Ferreira Franco W, Vinicios Borges Galdino M, RaineriCapeletti L, Helena Sberowsky B, Araújo Vieira R, César Figueiredo A, Müller Ramalho K, Alcântara Dos Santos FC, Francisco Biancardi M, de Marco P, Rubia Marques M (2020) Photobiomodulation and mandibular advancement modulates cartilage thickness and matrix deposition in the mandibular condyle. Photobiomodul Photomed Laser Surg 38(1):3-10

9. Fortier L, Chapman H, Pownder S, Roller B, Cross J, Cook J, Cole B (2016) BioCartilage improves cartilage repair compared with microfracture alone in an equine model of full-thickness cartilage loss. Am J Sports Med 44(9):2366-2374
10. Frisbie D, Oxford J, Southwood L, Trotter G, Rodkey W, Steadman R, Goodnight J, Mcllwraith W (2003) Early events in cartilage repair after subchondral bone microfracture. Clin Orthop Relat Res 407:215-227

11. Gigante A, Calcagno S, Cecconi S, Ramazzotti D, Manzotti S, Enea D (2011) Use of collagen scaffold and autologous bone marrow concentrate as a one-step cartilage repair in the knee: histological results of second-look biopsies at 1 year follow-up. Int J Immunopathol Pharmacol 24(1 Suppl 2):69-72

12. Gille J, Kunow J, Boisch L, Behrens P, Bos I, Hoffmann C, Köller W, Russlies M, Kurz B (2010) Cell-laden and cell-free matrix-induced chondrogenesis versus microfracture for the treatment of articular cartilage defects: a histological and biomechanical study in sheep. Cartilage 1(1):29-42

13. Han Y, Qu P, Zhang K, Bi Y, Zhou L, Xie D, Song H, Dong J, Qi J (2019) Storage solution containing hydrogen improves the preservation effect of osteochondral allograft. Cell Tissue Bank 20(2):201-208

14. Irwin RM, Bonassar $\amalg$, Cohen I, Matuska AM, Commins J, Cole B, Fortier LA (2019) The clot thickens: autologous and allogeneic fibrin sealants are mechanically equivalent in an ex vivo model of cartilage repair. PLoS One 14(11):e0224756

15. Jiang $X$, Huang $X$, Jiang $T$, Zheng $L$, Zhao J, Zhang $X$ (2018) The role of Sox 9 in collagen hydrogel-mediated chondrogenic differentiation of adult mesenchymal stem cells (MSCs). Biomater Sci 6(6):1556-1568

16. Kim JH, Heo JW, Lee DH (2020) Clinical and radiological outcomes after autologous matrix-induced chondrogenesis versus microfracture of the knee: a systematic review and metaanalysis with a minimum 2-year follow-up. Orthop J Sports Med 8(11):2325967120959280

17. Kurz B, Lemke AK, Fay J, Pufe T, Grodzinsky AJ, Schünke M (2005) Pathomechanisms of cartilage destruction by mechanical injury. Ann Anat 87(5-6):473-485

18. Lefebvre V, Dvir-Ginzberg M (2017) SOX9 and the many facets of its regulation in the chondrocyte lineage. Connect Tissue Res 58(1):2-14

19. Li S, Tallia F, Mohammed AA, Stevens MM, Jones JR (2020) Scaffold channel size influences stem cell differentiation pathway in 3-D printed silica hybrid scaffolds for cartilage regeneration. Biomater Sci. https://doi. org/10.1039/c9bm01829h

20. Nakamura S, Nakamura T, Kawahara H (2009) S-100 protein in human articular cartilage. Acta Orthop Scand 59(4):438-440

21. Schagemann J, Behrens P, Paech A, Riepenhof H, Kienast B, Mittelstädt $H$, Gille J (2018) Mid-term outcome of arthroscopic AMIC for the treatment of articular cartilage defects in the knee joint is equivalent to mini-open procedures. Arch Orthop Trauma Surg 138(6):819-825

22. Stanish WD, McCormack R, Forriol F, Mohtadi N, Pelet S, Desnoyers J, Restrepo A, Shive MS (2013) Novel scaffold-based BST-CarGel treatment results in superior cartilage repair compared with microfracture in a randomized controlled trial. J Bone Joint Surg Am 18:1640-1650

23. Reis AMS, Oliveira KP, de Paula IHF, da Silva AP, Tarragô JF, de Melo ON, Serakides R (2018) Nonlinear effects of caffeine on the viability, synthesis and gene expression of chondrocytes from the offspring of rats treated during pregnancy. Acta Histochem 120(6):505-512

24. Richter M, Zech S (2019) Matrix-associated stem cell transplantation (MAST) in chondral lesions at the ankle as part of a complex surgical approach- 5-year-follow-up in 100 patients. Foot Ankle Surg 25(3):264-271

25. Roca-Cusachs P, Sunyer R, Trepat X (2013) Mechanical guidance of cell migration: lessons from chemotaxis. Curr Opin Cell Biol 25(5):543-549

26. Pfeifer CG, Karl A, Kerschbaum M, Berner A, Lang S, Schupfner R, Koch M, Angele P, Nerlich M, Mueller MB (2019) TGF- $\beta$ signalling is suppressed under pro-hypertrophic conditions in MSC chondrogenesis due to TGF- $\beta$ receptor downregulation. Int I Stem Cells 12(1):139-150

27. Schüttler S, Andjelkov N (2012) Periosteal transplantation combined with the autologous matrix-induced chondrogenesis (AMIC) technique in isolated patellofemoral osteoarthritis: a case report. Cartilage 3(2):194-198

28. Sellam J, Berenbaum F (2010) The role of synovitis in pathophysiology and clinical symptoms of osteoarthritis. Nat Rev Rheumatol 6(11):625-635

29. Steadman J, Rodkey W, Rodrigo J (2001) Microfracture: surgical technique and rehabilitation to treat chondral defects. Clin Orthop Relat Res 391:S362-9

30. Vázquez-Portalatı N, Kilmer C, Panitch A, Liu J (2016) Characterization of collagen type I and II blended hydrogels for articular cartilage tissue engineering. Biomacromolecules 17(10):3145-3152 
31. Wong CC, Chen CH, Chan WP, Chiu LH, Ho WP, Hsieh FJ, Chen YT, Yang TL (2017) Single-stage cartilage repair using platelet-rich fibrin scaffolds with autologous cartilaginous grafts. Am J Sports Med 45(13):3128-3142

32. Xiao SP, Tang LS, Chen JY, Li ZT, Cheng GH, Chen QQ, Liu SH, Liu WG

(2019) Effect of cross-linked hyaluronate scaffold on cartilage repair: an in vivo study. Orthop Surg 11(4):679-689

33. Yan W, Xu X, Xu Q, Sun Z, Jiang Q, Shi D (2020) Platelet-rich plasma

combined with injectable hyaluronic acid hydrogel for porcine cartilage regeneration: a 6-month follow-up. Regen Biomater 7(1):77-90

\section{Submit your manuscript to a SpringerOpen ${ }^{\circ}$ journal and benefit from:}

- Convenient online submission

- Rigorous peer review

- Open access: articles freely available online

- High visibility within the field

- Retaining the copyright to your article

Submit your next manuscript at $\gg$ springeropen.com 